\title{
Clinical evaluation for the difference of absorbed doses calculated to medium and calculated to water by Monte Carlo method
}

\author{
Li Chen $^{1+}$, Botian Huang ${ }^{2 \dagger}$, Xiaoyan Huang ${ }^{1 *} \mathbb{D}$, Wufei Cao ${ }^{1}$, Wenzhao Sun ${ }^{1}$ and Xiaowu Deng ${ }^{1}$
}

\begin{abstract}
Background: To evaluate the difference of absorbed doses calculated to medium and to water by a Monte Carlo (MC) algorithm based treatment planning system (TPS), and to assess the potential clinical impact to dose prescription.

Methods: Thirty patients, 10 nasopharyngeal cancer (NPC), 10 lung cancer and 10 bone metastases cases, were selected for this study. For each case, the treatment plan was generated using a commercial MC based TPS and dose was calculated to medium $\left(D_{m}\right)$. The plan was recalculated for dose to water $\left(D_{w}\right)$ using the same Monitor Units (MU) and control points. The differences between $D_{m}$ and $D_{w}$ were qualitatively evaluated by dose-volume parameters and by the plan subtraction method. All plans were measured using the MapCheck2, and gamma passing rates were calculated.

Results: For NPC and Lung cases, the mean differences between $D_{w}$ and $D_{m}$ for the targets were less than $2 \%$ and the maximum difference was 3.9\%. The maximum difference of $\mathrm{D}_{2 \%}$ for the organs at risk (OARs) was 6.7\%. The maximum differences between $D_{w}$ and $D_{m}$ were as high as $10 \%$ in certain high density regions. For bone metastases cases, the mean differences between $D_{w}$ and $D_{m}$ for the targets were more than $2.2 \%$ and the maximum difference was $7.1 \%$. The differences between $D_{w}$ and $D_{m}$ for the OARs were basically negligible. At $3 \% \& 3 \mathrm{~mm}$ criterion, the gamma passing rate of $D_{w}$ plan and $D_{m}$ plan were close (>94\%).

Conclusion: The differences between $D_{w}$ and $D_{m}$ has little clinical impact for most clinical cases. In bony structures the differences may become clinically significant if the target/OAR is receiving doses close to its tolerance limit which can potentially influence the selection or rejection of a particular plan.
\end{abstract}

Keywords: Monte Carlo dose calculation, Absorbed dose to medium, Absorbed dose to water, Nasopharyngeal cancer, Gamma analysis

\section{Background}

Absorbed dose is an important parameter in characterizing the effect of radiation therapy for the efficacy of tumor eradication and protection from unacceptable damage to normal organs [1]. For historical reasons, in terms of dose, $D_{w}$ has been assumed for reporting the dose to various media. However, human body is not only composed of water. Many tissues in the body have

\footnotetext{
* Correspondence: huangxiaoy@sysucc.org.cn

${ }^{+}$Li Chen and Botian Huang contributed equally to this work.

'Department of Radiation Oncology, Sun Yat-Sen University Cancer Center, State Key Laboratory of Oncology in South China, Collaborative Innovation Center for Cancer Medicine, Guangdong Key Laboratory of Nasopharyngeal Carcinoma Diagnosis and Therapy, No. 651, Dongfeng Road East, Guangzhou 510060, China

Full list of author information is available at the end of the article
}

different densities than water, especially the bones and lung. For radiation therapy the dose absorbed to water cannot accurately represent the actual dose absorbed in different tissues. In practice, traditional treatment planning system (TPS) typically takes the effect of different tissue densities with attenuation and scatter into considerations but reports the dose at each location as the dose to water. Monte Carlo (MC) algorithm is the most accurate algorithm for dose calculation in that it simulates the transport properties of various particles in various media in the region of interest and scores the dose contribution locally to the medium with its assigned chemical composition as well as density. The resulting dose distributions may be different from those calculated by traditional dose calculation algorithms, especially for tissues of heterogeneity [2-4]. In 
recent years, $\mathrm{MC}$ has been increasingly adopted in clinical application [5-7]. There are a number of reasons for using $\mathrm{D}_{\mathrm{w}}$ for reporting of $\mathrm{MC}$ calculated doses. Two major ones are that it has been used in decades of clinical studies for outcome correlation with the dose, and that the calibration protocols are all referenced to water. A technical issue related to dose calibration is that an MC based TPS could model the chemical composition of various biological tissues by approximation as a function of Computed Tomography (CT) numbers based on data of the human body (reference International Commission on Radiation Units \& Measurements reports 44 and 46). Such an approximation may not perform well for non-biological materials like in a quality assurance (QA) phantom. MC based dose calculations typically report absorbed dose to media $\left(D_{m}\right)$. Therefore there is a need to convert between $D_{m}$ and $D_{w}$, and, as Siebers JV et al. [8] argued, MC is capable of doing the conversion. Siebers et al. presented a method to calculate the difference between $D_{m}$ and $D_{w}$ by applying the Bragg-Gray cavity theory, and their results showed a difference exceeding $10 \%$ in cortical bones.

Currently there is no consensus regarding whether $\mathrm{D}_{\mathrm{m}}$ or $D_{w}$ should be used for an MC based TPS $[9,10]$. When it comes to clinical application, the difference between $D_{w}$ and $D_{m}$ will affect interpretation of dose distribution and perhaps the value of prescription dose, leading to differences in plan evaluation, dose reporting, and dose verification. In this work, $D_{m}$ and $D_{w}$ were both calculated using Monaco TPS for 10 nasopharyngeal cancer (NPC) cases, 10 lung cancer cases and 10 bone target cases, in order to investigate the issue in two common clinical sites in which differences of dose distributions may be highlighted. Dose Volume Histogram $(\mathrm{DVH})$ was used to analyze dose parameters in the target and organ at risk (OAR), and three dimensional dose difference distributions between $\mathrm{D}_{\mathrm{m}}$ and $\mathrm{D}_{\mathrm{w}}$ were calculated. Gamma passing rates (measurement results vs $D_{m} / D_{w}$ plans) were calculated at different QA criteria to evaluate the dose accuracy.

\section{Methods}

\section{$D_{m}$ plan originally created for treatment}

Ten NPC cases in stage T3 or T4, 10 lung cancer cases and 10 bone target cases ( 7 cases of lumbar vertebra metastasis, 3 cases of thoracic vertebra metastasis) treated at Sun Yat-sen University Cancer Center were retrospectively chosen in this study. The gross tumor volumes (GTVs) and clinical tumor volume (CTV) were contoured by experienced radiation oncologists according to definitions in the ICRU 50 and ICRU 62 reports [11, 12], and the planning target volume (PTV) were generated following a set of physician prescribed margins that were consistent with departmental protocols specific to the disease sites. Monaco TPS (Version 5.0, Elekta) was used to create the treatment plans for step-and-shoot IMRT with an Elekta Synergy linac, and MC calculated $D_{m}$ was chosen for dose reporting. Nine equally spaced fields were used for NPC cases. The prescription of NPC cases and Lung cancer cases were 70 Gy (32 or 33 fractions, 5 days/week) and 65 Gy (26 fractions, 5 days/week) respectively. The main planning objectives for NPC are PTV $\mathrm{V}_{100 \%}>98 \%$ and PTV $\mathrm{V}_{110 \%}<10 \%\left(\mathrm{~V}_{\mathrm{x} \%}\right.$, is the percentage volume of reign of interest (ROI) that receives at least $\mathrm{x} \%$ prescription dose), spinal cord $\mathrm{D}_{2 \%}<45 \mathrm{~Gy}$, brain stem $\mathrm{D}_{2 \%}<54 \mathrm{~Gy}$, parotid gland $\mathrm{D}_{50 \%}<30 \mathrm{~Gy}$, optical nerve $\mathrm{D}_{2 \%}<54 \mathrm{~Gy}$, and the dose to lens as low as possible. For lung IMRT cases 5-7 fields were used. The planning objectives are PTV $\mathrm{V}_{100 \%}>95 \%$ and PTV $\mathrm{V}_{110 \%}<2 \%$, spinal cord $\mathrm{D}_{2 \%}<45 \mathrm{~Gy}$, normal lung $V_{20}$ Gy $<35 \%\left(V_{D}\right.$ Gy, is the percentage volume of ROI that receives at least absorbed dose D) and normal lung mean dose $<19 \mathrm{~Gy}$, heart $\mathrm{V}_{30 \mathrm{~Gy}}<40 \%$, and the maximum esophagus dose $<65 \mathrm{~Gy}$. For bone target cases, 5-7 fields were used. The prescription of bone target cases was 25 Gy (5Gy/fractions, 5 days/week). The main planning objectives are for PTV, $\mathrm{V}_{100 \%}>95 \%$ and $\mathrm{V}_{110 \%}<10 \%$, for spinal cord $\mathrm{D}_{\max }<26 \mathrm{~Gy}$, for lung $\mathrm{V}_{10 \mathrm{~Gy}}<15 \%$, and the maximum esophagus dose $<26 \mathrm{~Gy}$.

\section{$D_{w}$ calculation}

The MC algorithm in the Monaco TPS used for this study, called XVMC, calculates dose based upon mass density. A technical issue of dose calculation with $\mathrm{MC}$ in treatment planning is how to obtain the density and chemical composition data for the patient model from the CT. An approximation is made by assigning a voxel to certain type of tissue in the human body based on its Hounsfield unit (HU) in a certain range, and the mass density and composition data can be looked up in the International Commission on Radiation Units \& Measurements Reports No. 46 [13]. XVMC algorithm converts CT numbers to ED numbers using the user-defined CT-to-ED calibration table and takes with a fit function that maps continuously the electron density to mass density for matching a tissue with approximating cross section and attenuation coefficient data [14].

The conversion to $D_{w}$ can be calculated based on the distribution of $D_{m}$ plan according to the Bragg-Gray cavity theory:

$$
\mathrm{D}_{\mathrm{w}}=\mathrm{D}_{\mathrm{m}} s_{w, \text { med }}
$$

where $s_{w, m e d}$ is the mean unconstrained mass stop power ratio of water to media of primary electron spectrum, and $D_{w}$ is understood as the dose to the voxel replacement of water embedded to the actual media. Theoretically mass stop power ratio can be calculated by the following formula [8]: 


$$
s_{w, \text { med }}=\int_{0}^{E_{\max }}\left(\Phi_{E}\right)_{m}(S / \rho)_{w} d E / \int_{0}^{E_{\max }}\left(\Phi_{E}\right)_{m}(S / \rho)_{\operatorname{med}} d E
$$

where $(S / \rho)_{w}$ and $(S / \rho)_{\text {med }}$ are the unconstrained mass stop power of water and media, respectively. $\left(\Phi_{E}\right)_{m}$ is the primary electron fluence in the medium and $E_{\max }$ is the maximum energy in the $\left(\Phi_{E}\right)_{m}$ distribution. The stopping power ratio in Moncao was pre-calculated by approximation for tissue-like media.

The conversion from $D_{m}$ to $D_{w}$ in Monaco with a clinically accepted plan involved a simple recalculation with exactly the same set of plan parameters (all the geometric parameters and monitor units (MU)) retained. The stopping power ratios dependent of mass density were applied voxel by voxel. The matrix of dose calculation grid was $0.3 \mathrm{~cm} \times 0.3 \mathrm{~cm} \times 0.3 \mathrm{~cm}$, and the Monte Carlo statistical uncertainty was set at $3 \%$ per control point.

\section{$D_{m}$ and $D_{w}$ dose verification}

All the plans were measured with MapCHECK2 (Sun Nuclear, Florida, USA) to verify the dose distribution. MacpCHECK2 was mounted in a water-equivalent phantom (MapPHAN) with a $5 \mathrm{~cm}$ equivalent depth from the surface to the detectors. The TPS planed dose was calculated on the real phantom CT images without overriding the density. The measured dose distributions of composite fields were compared with the corresponding planned dose distributions $\left(\mathrm{D}_{\mathrm{m}}\right.$ or $\left.\mathrm{D}_{\mathrm{w}}\right)$, and the local dose normalization gamma $(\gamma)$ passing rates were calculated at the setting dose difference (DD) and distance to agreement (DTA). In order to eliminate dose in the out-of-field region where a large relative dose difference can be calculated and hence skew they result, a lower dose threshold (10\%) was set and below the threshold the $\gamma$ result was ignored. Using $3 \% \& 3 \mathrm{~mm}$, $2 \% \& 2 \mathrm{~mm}$ and $1 \% \& 1 \mathrm{~mm}$ tolerances, the gamma passing rates were calculated to find how the pass rates change with reduction of dose difference and DTA limits.

\section{Data analysis}

According to the ICRU 83 report, the volume-dose is recommended to describe the dose information in the ROIs, as $\mathrm{D}_{\mathrm{x} \%}$ to note the dose that $\mathrm{X} \%$ of volume of ROI receives [15]. For example, $D_{98 \%}$ means $98 \%$ of volume received the dose at specified value such as 65Gy. These DVH parameters were used for statistical analysis of $D_{w}$ and $D_{m}$ dose distributions. The bin width of the DVHs was $1 \mathrm{cGy}$, and the resolution for DVH sampling was $0.1 \mathrm{~cm}$. The difference between the $D_{w}$ and $D_{m}$ was calculated by:

$$
\operatorname{Diff}(\%)=\left(\left(D_{\mathrm{x}} \%\right)_{\mathrm{w}}-\left(\mathrm{D}_{\mathrm{x} \%}\right)_{\mathrm{m}}\right) /\left(\mathrm{D}_{\mathrm{x} \%}\right)_{\mathrm{w}} \times 100
$$

The plan subtraction method was used to evaluate the spatial dose difference distribution of $\mathrm{D}_{\mathrm{w}}$ and $\mathrm{D}_{\mathrm{m}}$.

Paired t-tests were performed using the SPSS software (Version 19, SPSS, Inc., USA) to determine the statistical significance of the difference between $\mathrm{D}_{\mathrm{w}}$ and $\mathrm{D}_{\mathrm{m}}$, with a $p$-value $<0.05$ as the threshold for consideration as statistically significant.

\section{Results}

\section{$D_{w}$ and $D_{m}$ for NPC cases}

Figure 1 shows the comparison of the DVH results with $D_{w}$ and $D_{m}$ for a typical NPC treatment plan. There were small but systematic deviations from $D_{m}$ to $D_{w}$ in the planning target volumes (PTVs). Table 1 shows the

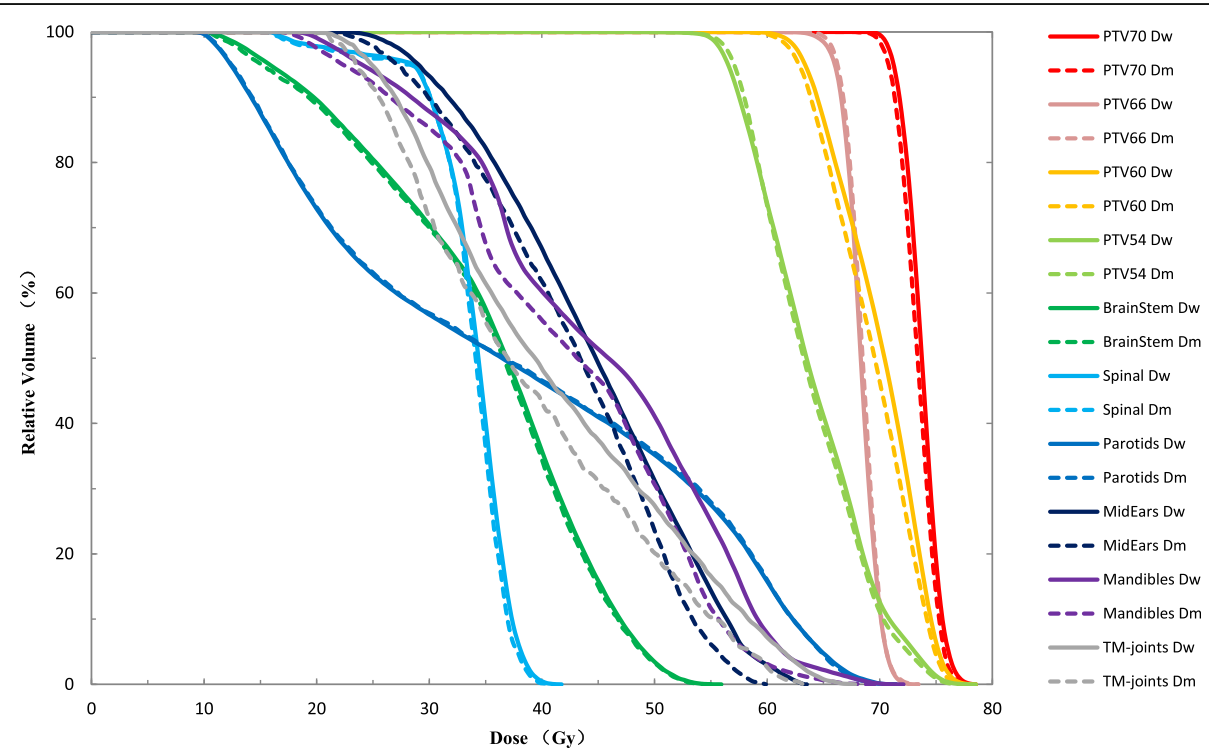

Fig. 1 DVH comparison for $D_{w}$ and $D_{m}$ results from the MC-based Monaco TPS for a typical NPC case 
mean and difference in dose-volume indices calculated with MC, evaluated for 10 NPC cases. Except for the $\mathrm{D}_{50 \%}$ and $\mathrm{D}_{2 \%}$ of PTV66, and $\mathrm{D}_{98 \%}$ of PTV54, all DVH indices for all PTVs were different with statistical significance $(p<0.05)$, including $\mathrm{D}_{98 \%}, \mathrm{D}_{50 \%}$, and $\mathrm{D}_{2 \%}\left(\mathrm{D}_{\mathrm{x} \%}\right.$, the minimum dose that $\mathrm{x} \%$ of the volume of the organ receives from the cumulative $\mathrm{DVH}$ ). The possible reason for PTV66 behaved differently from the others may be that PTV66 is the lymph gland target, small in size and relatively variable in location among different patients. For the $\mathrm{D}_{2 \%}$ of PTV70, PTV66, PTV60 and PTV54, the

Table 1 The mean and standard deviation of $D_{w}$ and $D_{m}$ in dose-volume indices calculated with Monte Carlo for 10 NPC IMRT cases

\begin{tabular}{|c|c|c|c|c|c|}
\hline $\mathrm{ROI}$ & Parameter & $\mathrm{D}_{\mathrm{w}}(\mathrm{Gy})$ & $D_{m}(G y)$ & Diff(\%) & $p$ \\
\hline \multirow[t]{3}{*}{ PTV70 } & $D_{98 \%}$ & $70.7 \pm 0.6$ & $70.2 \pm 0.3$ & $0.7 \pm 0.5$ & 0.002 \\
\hline & $D_{50 \%}$ & $74.3 \pm 0.5$ & $73.6 \pm 0.3$ & $0.9 \pm 0.4$ & $<0.001$ \\
\hline & $D_{2 \%}$ & $78.2 \pm 1.5$ & $76.7 \pm 1.0$ & $1.9 \pm 1.1$ & 0.001 \\
\hline \multirow[t]{3}{*}{ PTV66 } & $D_{98 \%}$ & $64.6 \pm 2.1$ & $65.0 \pm 2.2$ & $-0.6 \pm 0.4$ & 0.002 \\
\hline & $D_{50 \%}$ & $69.0 \pm 0.6$ & $69.0 \pm 0.6$ & $0.1 \pm 0.3$ & 0.923 \\
\hline & $\mathrm{D}_{2 \%}$ & $72.4 \pm 1.2$ & $72.1 \pm 1.5$ & $0.4 \pm 1.0$ & 0.235 \\
\hline \multirow[t]{3}{*}{ PTV60 } & $D_{98 \%}$ & $63.2 \pm 1.1$ & $62.7 \pm 1.0$ & $0.7 \pm 0.5$ & 0.001 \\
\hline & $D_{50 \%}$ & $71.7 \pm 0.9$ & $71.1 \pm 0.9$ & $0.9 \pm 0.3$ & $<0.001$ \\
\hline & $\mathrm{D}_{2 \%}$ & $77.4 \pm 1.3$ & $76.1 \pm 0.9$ & $1.7 \pm 1.0$ & $<0.001$ \\
\hline \multirow[t]{3}{*}{ PTV54 } & $D_{98 \%}$ & $56.4 \pm 0.7$ & $56.6 \pm 0.5$ & $0.2 \pm 0.4$ & 0.144 \\
\hline & $D_{50 \%}$ & $65.0 \pm 1.2$ & $64.7 \pm 1.2$ & $0.6 \pm 0.3$ & $<0.001$ \\
\hline & $D_{2 \%}$ & $76.1 \pm 1.2$ & $75.1 \pm 0.9$ & $1.3 \pm 0.7$ & $<0.001$ \\
\hline \multirow[t]{2}{*}{ Spinal Cord } & $D_{50 \%}$ & $34.0 \pm 1.6$ & $33.9 \pm 1.7$ & $0.5 \pm 0.3$ & $<0.001$ \\
\hline & $D_{2 \%}$ & $39.6 \pm 1.2$ & $39.2 \pm 1.2$ & $0.8 \pm 0.3$ & $<0.001$ \\
\hline \multirow[t]{2}{*}{ Brain Stem } & $D_{50 \%}$ & $38.2 \pm 2.3$ & $38.1 \pm 2.2$ & $0.4 \pm 0.3$ & 0.002 \\
\hline & $D_{2 \%}$ & $57.3 \pm 6.8$ & $57.1 \pm 6.8$ & $0.3 \pm 0.2$ & $<0.001$ \\
\hline \multirow[t]{2}{*}{ Parotids } & $D_{50 \%}$ & $40.9 \pm 7.1$ & $41.0 \pm 7.0$ & $-0.1 \pm 0.6$ & 0.901 \\
\hline & $D_{2 \%}$ & $69.3 \pm 1.6$ & $69.2 \pm 1.6$ & $0.2 \pm 0.3$ & 0.136 \\
\hline \multirow[t]{2}{*}{ Lens } & $D_{50 \%}$ & $4.4 \pm 1.9$ & $4.4 \pm 1.9$ & $0.7 \pm 0.9$ & 0.019 \\
\hline & $\mathrm{D}_{2 \%}$ & $6.2 \pm 2.8$ & $6.2 \pm 2.8$ & $0.2 \pm 0.6$ & 0.082 \\
\hline \multirow[t]{2}{*}{ Optic nerves } & $D_{50 \%}$ & $35.9 \pm 21.4$ & $35.5 \pm 21.5$ & $1.6 \pm 4.4$ & 0.097 \\
\hline & $\mathrm{D}_{2 \%}$ & $54.1 \pm 23.7$ & $53.7 \pm 23.4$ & $0.4 \pm 0.8$ & 0.078 \\
\hline \multirow[t]{2}{*}{ TM-Joints } & $D_{50 \%}$ & $44.2 \pm 6.4$ & $42.0 \pm 6.0$ & $5.1 \pm 0.7$ & $<0.001$ \\
\hline & $D_{2 \%}$ & $67.2 \pm 4.3$ & $64.6 \pm 4.2$ & $4.5 \pm 1.2$ & $<0.001$ \\
\hline \multirow[t]{2}{*}{ Mid-Ears } & $D_{50 \%}$ & $43.3 \pm 4.1$ & $42.4 \pm 3.7$ & $2.1 \pm 1.7$ & 0.009 \\
\hline & $D_{2 \%}$ & $64.2 \pm 4.8$ & $62.0 \pm 5.0$ & $3.4 \pm 1.7$ & $<0.001$ \\
\hline \multirow[t]{2}{*}{ Mandibles } & $D_{50 \%}$ & $49.5 \pm 6.8$ & $46.8 \pm 7.2$ & $5.5 \pm 1.8$ & $<0.001$ \\
\hline & $\mathrm{D}_{2 \%}$ & $67.4 \pm 4.4$ & $64.2 \pm 4.7$ & $4.8 \pm 1.5$ & $<0.001$ \\
\hline \multirow[t]{2}{*}{ Temporal lobe } & $D_{50 \%}$ & $16.8 \pm 7.3$ & $16.7 \pm 7.3$ & $0.6 \pm 0.7$ & 0.003 \\
\hline & $D_{2 \%}$ & $64.2 \pm 6.0$ & $63.6 \pm 6.0$ & $0.9 \pm 0.3$ & $<0.001$ \\
\hline \multirow[t]{2}{*}{ Tongue } & $D_{50 \%}$ & $47.7 \pm 6.7$ & $47.4 \pm 6.7$ & $0.6 \pm 0.3$ & $<0.001$ \\
\hline & $D_{2 \%}$ & $65.3 \pm 5.3$ & $65.2 \pm 5.5$ & $0.2 \pm 0.6$ & 0.340 \\
\hline
\end{tabular}

values of the $D_{m}$ plan are less than that of $D_{w}$, and the mean deviation was $1.9 \pm 1.1 \%, 0.4 \pm 1.0 \%, 1.7 \pm 1.0 \%$ and $1.3 \pm 0.7 \%$, respectively. The difference between $D_{w}$ and $D_{m}$ in the mean dose of PTVs were within $1 \%$.

As for the OARs, the $\mathrm{D}_{50 \%}$ increased when $\mathrm{D}_{\mathrm{m}}$ was converted to $D_{w}$, and this was a statistically significant result except for the optic nerve and parotid gland. The median dose of T-M joints and mandibular in the $\mathrm{D}_{\mathrm{m}}$ plans were at least $5 \%$ less than that in the $\mathrm{D}_{\mathrm{w}}$ plans. The $\mathrm{D}_{2 \%}$ of spinal cord, brain stem, parotid gland, lens, optic nerves, temporal lobe, and tongue increased by less than $1 \%$ from $D_{m}$ to $D_{w}$. However, the $\mathrm{D}_{2 \%}$ of T-M joints and mandibular suffered about $5 \%$ change from $D_{m}$ to $D_{w}$.

\section{$D_{w}$ and $D_{m}$ for lung cancer cases}

Figure 2 shows that, for lung cancer cases, the difference between $D_{w}$ and $D_{m}$ is less obvious than in the NPC cases. Table 2 shows that the $\mathrm{D}_{2 \%}$ of PTV65 and the $\mathrm{D}_{98 \%}$ of PTV50 were statistically significant $(p<0.05)$, and the mean deviation were $0.3 \pm 0.4 \%$ and $-0.3 \pm 0.3 \%$, respectively. There were no other statistically significant differences for other DVH indices evaluated for PTVs. All deviations were with $1 \%$. For the OARs, the median dose $\mathrm{D}_{50 \%}$ of spinal cord and heart were slightly increased from $D_{m}$ to $D_{w}$ with the mean deviation at $0.3 \pm 0.3 \%$ and $1.1 \pm$ $0.5 \%$, respectively, and this was statistically significant. There were no statistically significant differences between $D_{w}$ and $D_{m}$ in lung and esophagus. For the $D_{2 \%}$ of spinal cord, lung, esophagus and heart, there were statistically significant differences between $\mathrm{D}_{\mathrm{w}}$ and $\mathrm{D}_{\mathrm{m}}$, and the mean deviation were $0.3 \pm 0.4 \%,-0.6 \pm 0.5 \%,-0.7 \pm 0.5 \%$, and $0.6 \pm 0.6 \%$, respectively. All the differences in the DVH indices evaluated were within $2 \%$.

\section{$D_{w}$ and $D_{m}$ for bone target cases}

Figure 3 shows that, for bone metastases cases, the differences between $D_{w}$ and $D_{m}$ for PTV targets are more obvious than those in the NPC cases and lung cases. From Table 3, all DVH indices for the PTVs were different with statistical significance $(p<0.01)$. The $\mathrm{D}_{98 \%}$, $\mathrm{D}_{50 \%}$, and $\mathrm{D}_{2 \%}$ deviation of PTV25 were $3.0 \pm 1.2 \%, 3.5$ $\pm 1.4 \%$ and $4.4 \pm 1.9 \%$, respectively. For the PTV20, $\mathrm{D}_{98 \%}, \mathrm{D}_{50 \%}$, and $\mathrm{D}_{2 \%}$ deviations were $2.2 \pm 0.7 \%, 2.8 \pm$ $0.7 \%$ and $3.8 \pm 1.7 \%$, respectively. There were basically negligible differences between $D_{w}$ and $D_{m}$ in spinal, lung and esophagus. All the differences in the DVH indices evaluated for OARs were within $0.6 \%$.

\section{Dose difference distribution maps}

By subtracting the re-calculated $D_{w}$ plan and original $\mathrm{D}_{\mathrm{m}}$ plans, the dose difference of three-dimensional distribution can be obtained. The dose difference (diff) is defined by diff $(\%)=\left(D_{w}-D_{m}\right) / D_{p} \times 100$, where $D_{p}$ is the prescription dose. Figure 4 shows the difference 


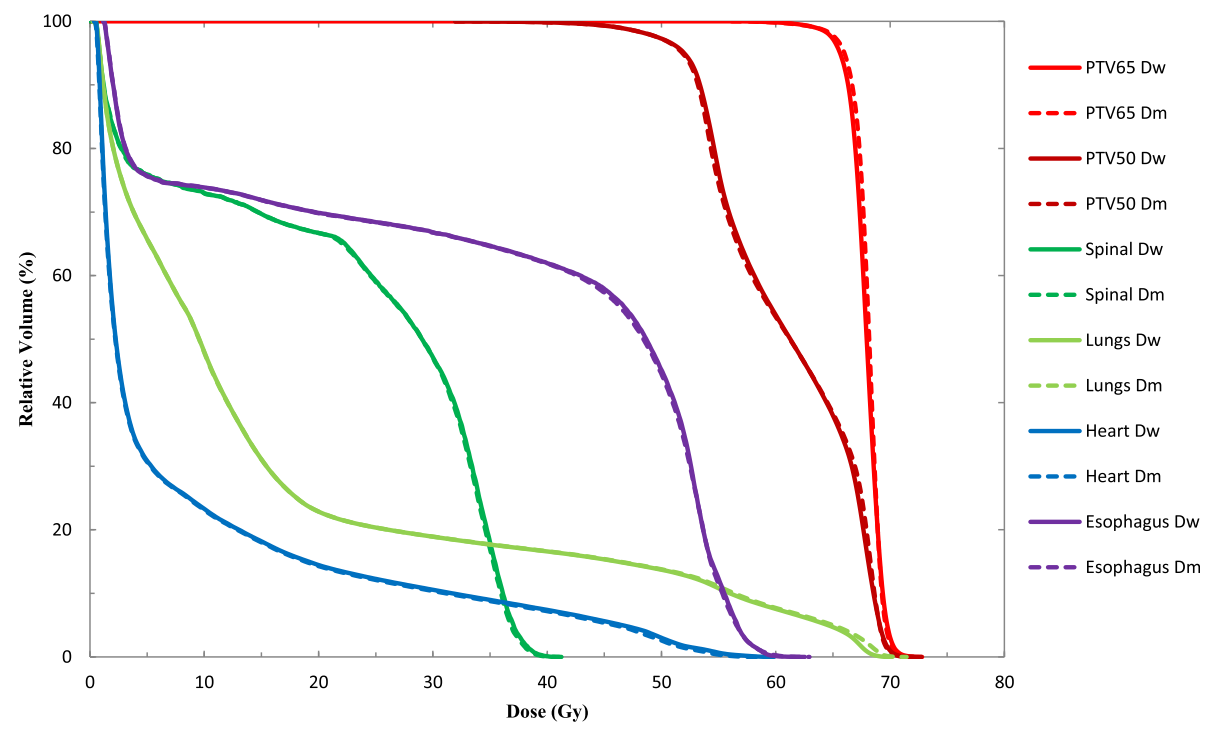

Fig. 2 DVH comparison for $D_{w}$ and $D_{m}$ results from the MC-based Monaco TPS for a typical Lung case

distribution in three-dimensions of a typical NPC case between $D_{w}$ and $D_{m}$. A typical case of lung cancer is shown in Fig. 5 and a case of bone metastasis is shown in Fig. 6. The blue to purple gradient legend represented the dose difference values ranging from 0 to $10 \%$. It can be seen from Fig. 4 and Fig. 5 that the difference between $D_{w}$ and $D_{m}$ could be higher than $5 \%$ in bone, while the differences between $D_{w}$ and $D_{m}$ in soft tissues were less obvious (usually smaller than 3\%). From Fig. 6 the differences between $D_{w}$ and $D_{m}$ in thoracic vertebra bone were about $3-8 \%$, a little lower than the result in

Table 2 The mean and standard deviation of $D_{w}$ and $D_{m}$ in dose-volume indices calculated with Monte Carlo for 10 Lung IMRT cases

\begin{tabular}{llllll}
\hline ROI & Parameter & $D_{w}(G y)$ & $D_{m}(G y)$ & Diff(\%) & $p$ \\
\hline PTV65 & $D_{98 \%}$ & $60.7 \pm 2.9$ & $60.6 \pm 2.9$ & $-0.2 \pm 0.5$ & 0.274 \\
& $D_{50 \%}$ & $68.1 \pm 0.3$ & $68.3 \pm 0.3$ & $-0.3 \pm 0.3$ & 0.106 \\
& $D_{2 \%}$ & $71.1 \pm 0.9$ & $70.9 \pm 1.0$ & $0.3 \pm 0.4$ & 0.032 \\
PTV50 & $D_{98 \%}$ & $49.6 \pm 1.0$ & $49.8 \pm 1.0$ & $-0.3 \pm 0.3$ & 0.004 \\
& $D_{50 \%}$ & $64.2 \pm 4.2$ & $64.2 \pm 4.3$ & $-0.1 \pm 0.4$ & 0.707 \\
& $D_{2 \%}$ & $70.8 \pm 1.0$ & $70.6 \pm 1.1$ & $0.2 \pm 0.4$ & 0.137 \\
Spinal & $D_{50 \%}$ & $28.1 \pm 9.8$ & $28.1 \pm 9.7$ & $0.3 \pm 0.3$ & 0.001 \\
& $D_{2 \%}$ & $41.2 \pm 2.4$ & $41.1 \pm 2.4$ & $0.3 \pm 0.4$ & 0.046 \\
Lungs & $D_{50 \%}$ & $8.5 \pm 2.9$ & $8.5 \pm 2.9$ & $-0.2 \pm 0.2$ & 0.052 \\
& $D_{2 \%}$ & $65.8 \pm 3.9$ & $66.2 \pm 4.1$ & $-0.6 \pm 0.5$ & 0.003 \\
Esophagus & $D_{50 \%}$ & $40.0 \pm 16.9$ & $40.0 \pm 16.9$ & $-0.1 \pm 0.6$ & 0.718 \\
& $D_{2 \%}$ & $60.2 \pm 2.9$ & $60.7 \pm 3.1$ & $-0.7 \pm 0.5$ & 0.004 \\
Heart & $D_{50 \%}$ & $6.1 \pm 7.0$ & $6.1 \pm 7.0$ & $1.1 \pm 0.5$ & 0.010 \\
& $D_{2 \%}$ & $51.0 \pm 10.7$ & $50.5 \pm 10.5$ & $0.6 \pm 0.6$ & 0.001 \\
\hline
\end{tabular}

head bone in Fig. 3. It's probably because the bone density of the thoracic vertebra is different from that of the head bone.

\section{Dose verification}

At normal QA criterion, 3\% dose difference and $3 \mathrm{~mm}$ distance to agreement, the gamma pass rates of $D_{w}$ plan and $D_{m}$ plan are all above $94 \%$ and very close. But when the tolerances become stricter, the gamma passing rates decreases dramatically, and $D_{w}$ plans gamma pass rates become better than the $D_{m}$ plans (Table 4).

\section{Discussions}

With the application of MC algorithm for dose calculation in radiation therapy, whether the dose should be calculated to medium or to water has been an unsettled debate $[9,10$, 16]. The arguments that support $D_{w}$ include that beam data was measured in water, that the beam output was calibrated in water, and that most clinical experience were based on dose to water, etc. However, the compelling argument to support the use of $D_{m}$ is that it represents the true dose at each location of specific medium. It is the unique advantage of Monte Carlo in that $\mathrm{D}_{\mathrm{m}}$ can be calculated directly, but $D_{m}$ to $D_{w}$ using stopping power ratios may involve an uncertainty [17]. In reality, different TPS use different dose calculation algorithms to produce $D_{w}$, from direct calculation to applying conversion factors. According to the AAPM TG 105 report [18], when the element components are considered in dose calculation, both $\mathrm{D}_{\mathrm{m}}$ and $D_{w}$ should be available for evaluation. When comes to a specific clinical situation, the difference between $D_{m}$ and $D_{w}$ should be known. N Dogan et al. [19] showed that converting $D_{m}$ to $D_{w}$ in EGS4 MC-calculated IMRT treatment 


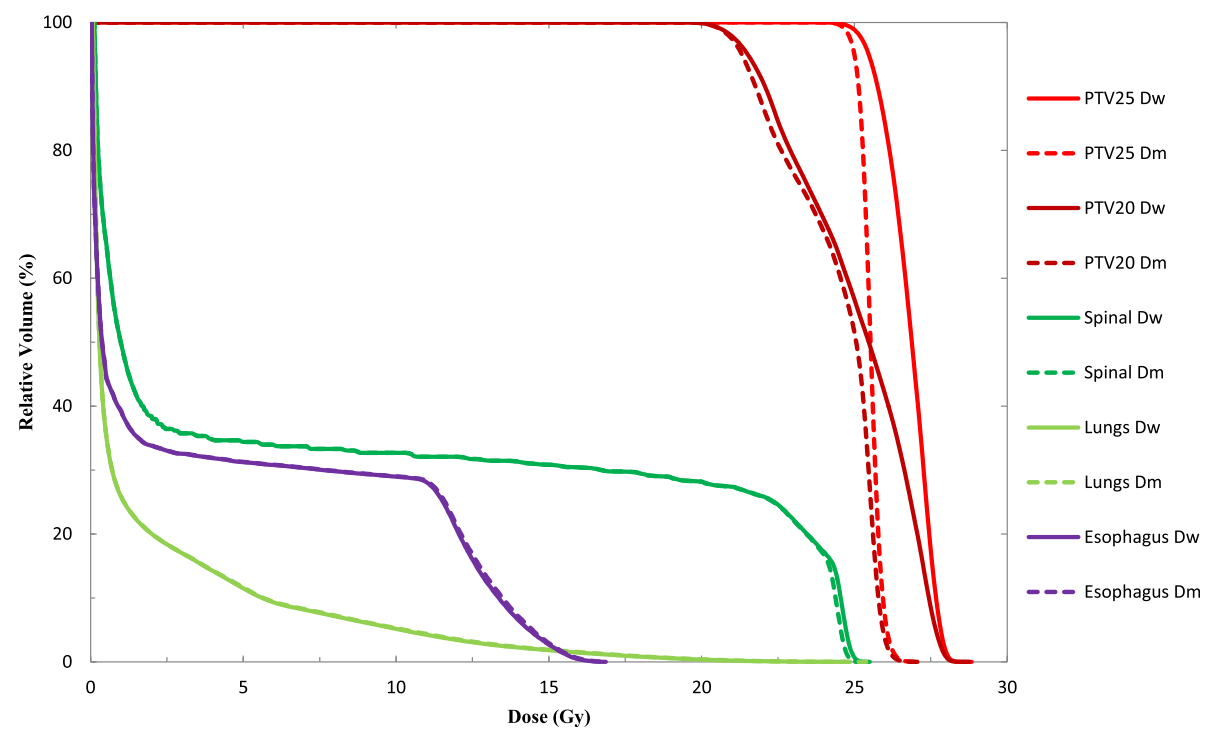

Fig. 3 DVH comparison for $D_{w}$ and $D_{m}$ results from the MC-based Monaco TPS for a typical thoracic vertebra metastasis of prostate cancer case

plans introduces a systematic error in target and critical structure DVHs, and this systematic error may reach up to $5.8 \%$ for $\mathrm{H} \& \mathrm{~N}$ and $8.0 \%$ for prostate cases when the hard-bone-containing structures such as femoral heads are present.

From our work using Monaco for NPC and lung cancer, $D_{m}$ was less than $D_{w}$. The mean deviation for soft tissues was within $2 \%$. For T-M joints and mandibular, the mean deviation was greater than $5 \%$, and in regions of unspecified normal bone the difference could reach $10 \%$. Our results agreed nicely with the work by Siebers et al. [8]. It is interesting to find, based on our study, that there was hardly any difference between $D_{w}$ and $\mathrm{D}_{\mathrm{m}}$ in low density regions. Although the stopping

Table 3 The mean and standard deviation of $D_{w}$ and $D_{m}$ in dose-volume indices calculated with Monte Carlo for 10 bone target cases

\begin{tabular}{llllll}
\hline ROI & Parameter & $D_{w}(G y)$ & $D_{m}(G y)$ & Diff(\%) & $p$ \\
\hline PTV25 & $D_{98 \%}$ & $25.7 \pm 0.9$ & $24.9 \pm 1.0$ & $3.0 \pm 1.2$ & 0.002 \\
& $D_{50 \%}$ & $27.2 \pm 0.3$ & $26.2 \pm 0.4$ & $3.5 \pm 1.4$ & $<0.001$ \\
& $D_{2 \%}$ & $28.2 \pm 0.4$ & $27.0 \pm 0.4$ & $4.4 \pm 1.9$ & $<0.001$ \\
PTV20 & $D_{98 \%}$ & $21.6 \pm 0.9$ & $21.1 \pm 1.0$ & $2.2 \pm 0.7$ & 0.019 \\
& $D_{50 \%}$ & $25.2 \pm 1.7$ & $24.4 \pm 1.7$ & $2.8 \pm 0.7$ & $<0.001$ \\
& $D_{2 \%}$ & $27.9 \pm 0.4$ & $26.8 \pm 0.3$ & $3.8 \pm 1.7$ & $<0.001$ \\
Spinal & $D_{50 \%}$ & $14.4 \pm 9.9$ & $14.3 \pm 9.9$ & $0.4 \pm 0.5$ & 0.025 \\
& $D_{2 \%}$ & $24.4 \pm 1.4$ & $24.3 \pm 1.3$ & $0.5 \pm 0.3$ & 0.001 \\
Lungs & $D_{50 \%}$ & $1.4 \pm 1.4$ & $1.4 \pm 1.4$ & $0.0 \pm 0.3$ & 0.999 \\
& $D_{2 \%}$ & $14.2 \pm 5.7$ & $14.3 \pm 5.7$ & $-0.6 \pm 0.6$ & 0.011 \\
Esophagus & $D_{50 \%}$ & $5.1 \pm 6.6$ & $5.1 \pm 6.6$ & $-0.6 \pm 1.0$ & 0.950 \\
& $D_{2 \%}$ & $21.0 \pm 3.6$ & $21.0 \pm 3.6$ & $0.1 \pm 0.4$ & 0.453 \\
\hline
\end{tabular}

power ratio for both cortical bone and air can be above 1.10, the stopping power ratio is close to 1 for low density tissues like lung. For this reason, the issue with using $\mathrm{D}_{\mathrm{w}}$ or $\mathrm{D}_{\mathrm{m}}$ may have a minimal effect for majority of clinical situations.

The dose difference between $\mathrm{D}_{\mathrm{w}}$ and $\mathrm{D}_{\mathrm{m}}$ in bony structures may become clinically significant if the OAR is receiving doses close to its tolerance dose limit which can influence selection or rejection of a particular plan. The dose calculated by MC may need to be carefully evaluated in certain situations, e.g. bone metastasis, bone tumor, or constraining a hot spot in bone that becomes a limiting factor in plan optimization. From the Fig. 3, for PTV of the bone target cases, though the target dose coverages (the target volume (\%) received the prescription dose) of $D_{m}$ and converted $D_{w}$ plan were similar, the mean median dose of $\mathrm{D}_{\mathrm{w}}$ plan increased by $3.5 \%$ comparing with that of $\mathrm{D}_{\mathrm{m}}$ plan (Table 3). That means the dose prescription for bone target could be about 3.5\% higher than that of using $\mathrm{D}_{\mathrm{w}}$ dose, and their treatment response and outcome may need further study in the future.

Previous studies [16, 20] using EGS4/MCSIM Monte Carlo and AXB dose calculations proved that conventional model based algorithms predicted dose distributions in bone that were closer to $D_{m}$ distributions than to $D_{w}$ distributions. It is therefore better to use $D_{m}$ for consistency with previous radiation therapy experience. Our measurements showed that at widely used reference standard, 3\% dose difference and $3 \mathrm{~mm}$ DTA, the $D_{m}$ and $D_{w}$ plan gamma passing rates were very close, but when the gamma calculation standard became stricter, the $D_{w}$ was closer to the result of measurement than the $D_{m}$. That's because the MapCheck2 CT images without forcing 


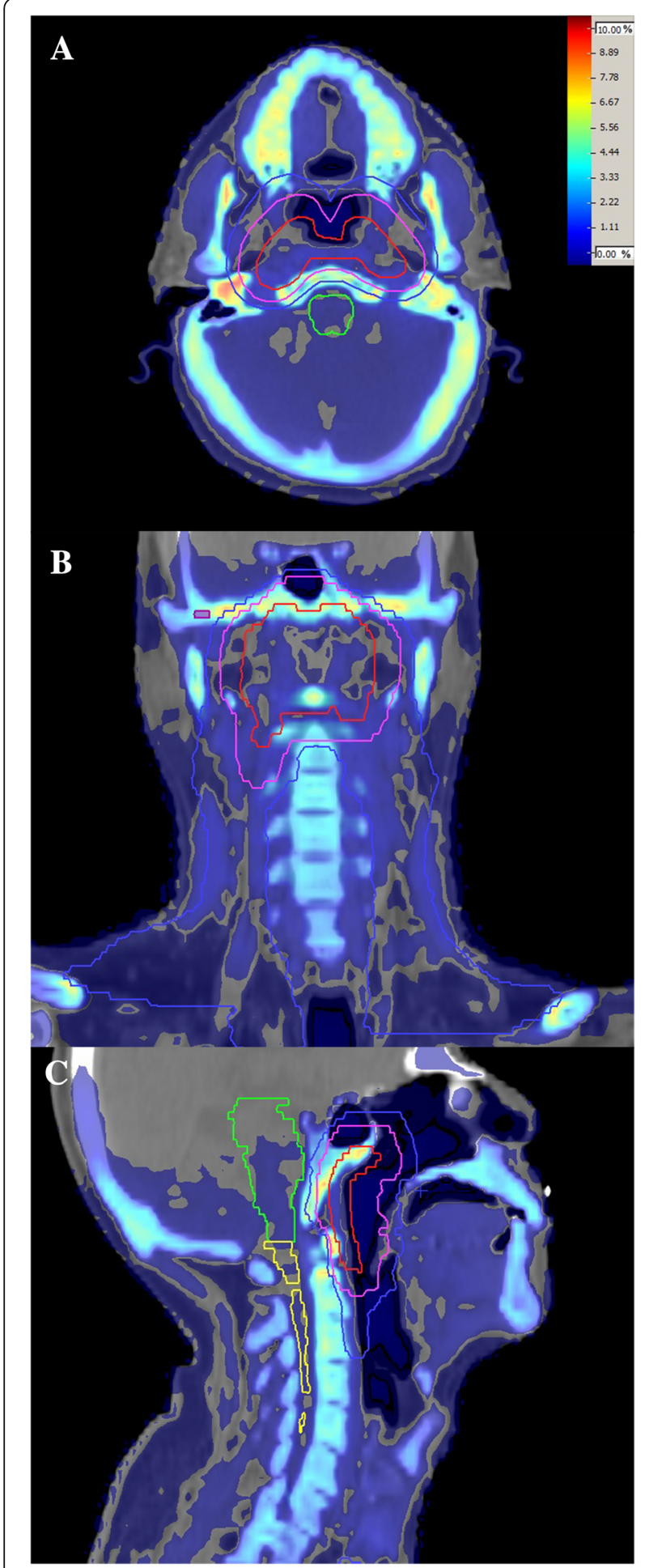

Fig. $4 D_{w}$ and $D_{m}$ dose difference map displayed in axial (a), coronal (b), and sagittal (c) slices in a typical NPC case

density were used to calculate the planned dose distribution, where the MapCheck2 detectors are made of high density metallic elements and the detectors are always calibrated by $D_{w}$. The CT scanner used for acquisition of

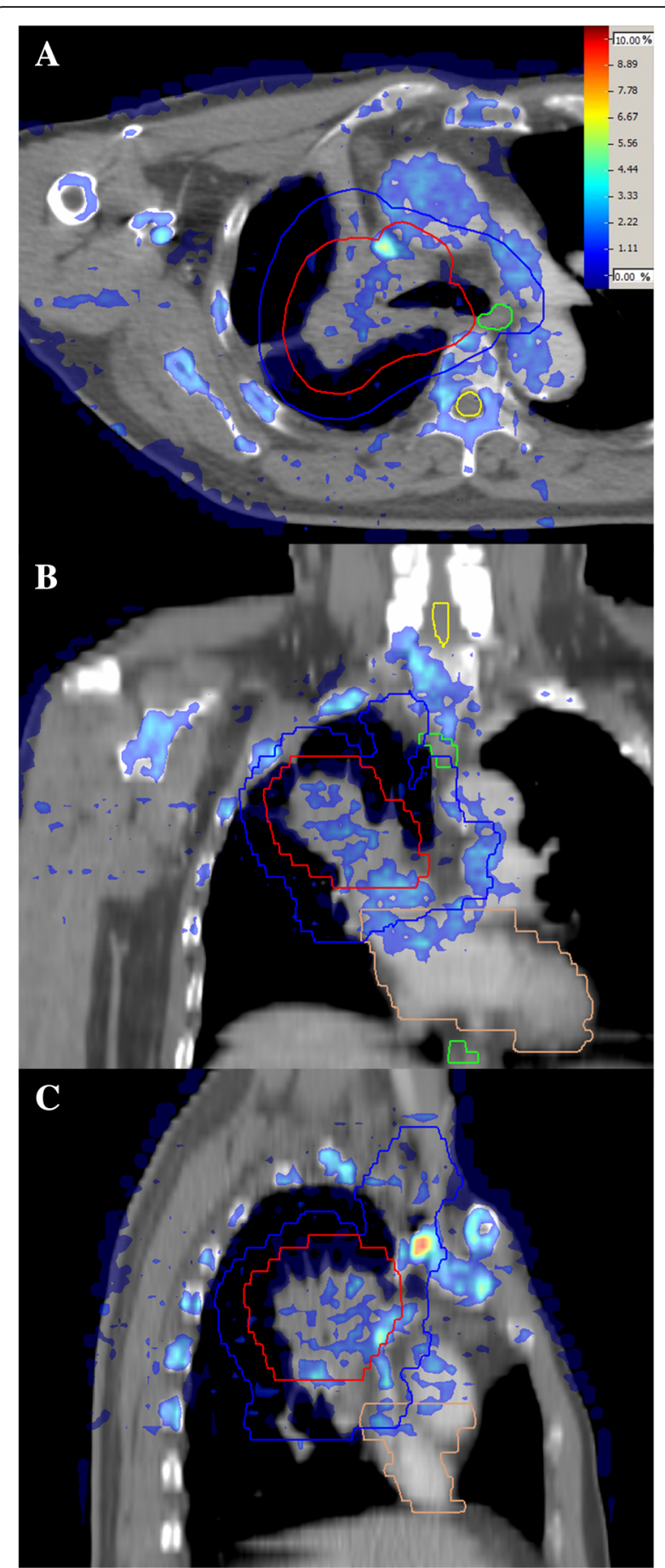

Fig. $5 D_{w}$ and $D_{m}$ dose difference map displayed in axial (a), coronal (b), and sagittal (c) slices in a typical lung case

patient simulation images has the limitation of scanning high density material such as the diode and the TPS also has limitation while accepting CT images with high density material. In our practice, $\mathrm{D}_{\mathrm{m}}$ is used for treatment planning, and physicians and physicists will be consulted 


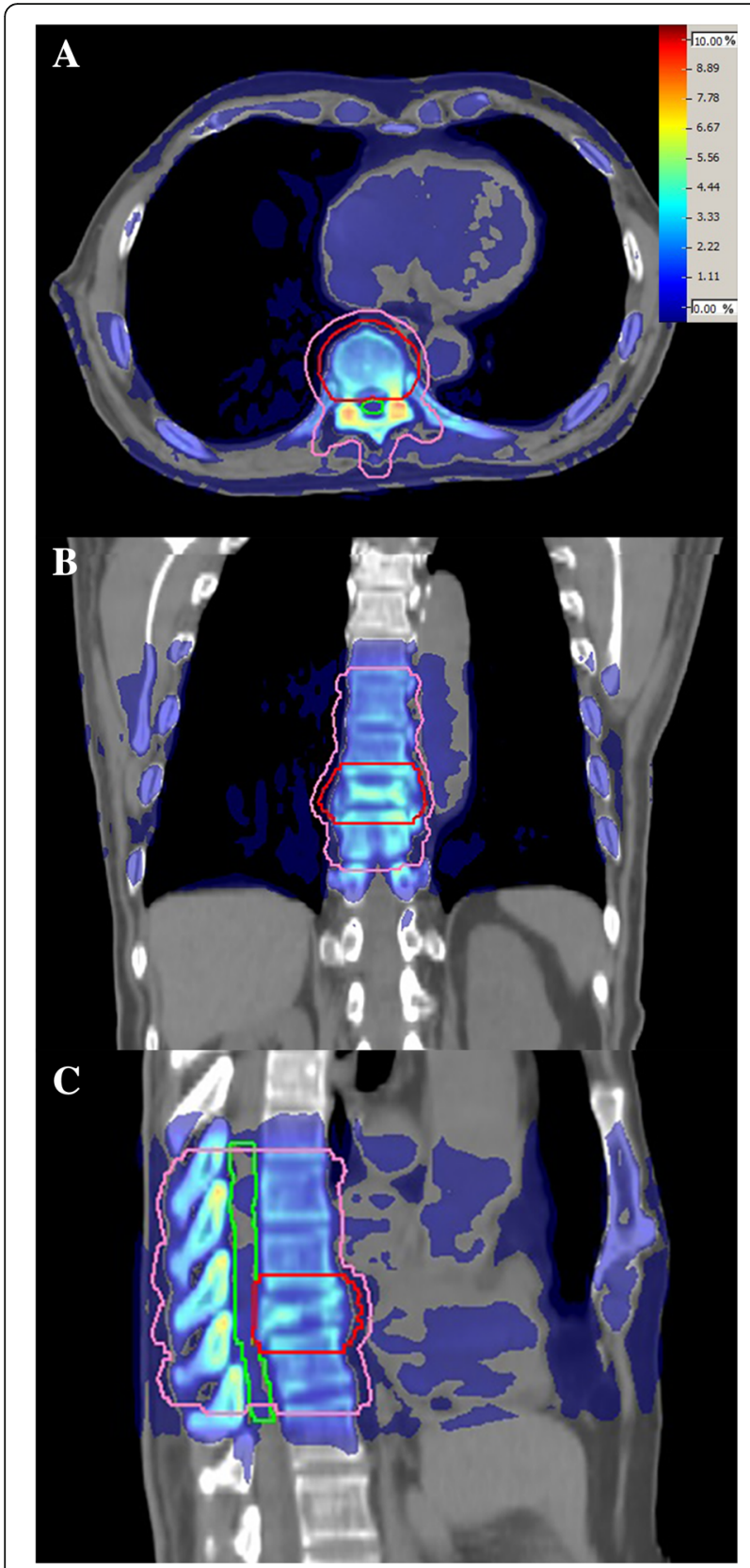

Fig. $6 D_{w}$ and $D_{m}$ dose difference map displayed in axial (a), coronal (b), and sagittal (c) slices in a typical bone target case
Table 4 The local gamma passing percentages at different quality assurance criteria for NPC IMRT cases

\begin{tabular}{lllll}
\hline Tolerance & Measurement vs $D_{m}$ & Measurement vs $D_{w}$ & $t$ & $p$ \\
\hline $3 \% \& 3 \mathrm{~mm}$ & $94.3 \pm 3.2 \%$ & $97.1 \pm 2.2 \%$ & -2.464 & 0.036 \\
2\%\&2 mm & $79.1 \pm 2.7 \%$ & $89.1 \pm 1.6 \%$ & -2.882 & 0.018 \\
$1 \% \& 1 \mathrm{~mm}$ & $43.6 \pm 2.6 \%$ & $56.1 \pm 2.3 \%$ & -3.024 & 0.014 \\
\hline
\end{tabular}

$\mathrm{t}, p$ values were calculated by paired t-tests using the SPSS 19.0 in case conversion to $\mathrm{D}_{\mathrm{w}}$ in bone may affect the decisions to choose the appropriate dose distribution for treatment.

Conversion to $D_{w}$ may be necessary for dose verification in the quality assurance phantom. If a water phantom is used, the difference between $D_{m}$ and $D_{w}$ can be ignored. Kan MW et al. [20] showed that for a heterogeneous phantom with high density materials contained the difference between $D_{m}$ and $D_{w}$ has an effect on the passing rate of QA measurement. Our results (Table 4) showed there were obvious differences between the $D_{m}$ and $D_{w}$ plan gamma passing rates when the QA criteria became strict. A simple method to bypass the problem is to assign a uniform density to the phantom and calculate to either $D_{m}$ or $D_{w}$ in a consistent manner. The choice of an appropriate density needs to be validated by an independent method such as point dose measurement.

\section{Conclusions}

Overall, the dose differences between $D_{m}$ and $D_{w}$ calculated by $\mathrm{MC}$ algorithm in Monaco are small in regions that have densities close or low to water. Our results show that dose calculated to medium by Monaco can be used clinically. In high density regions like cortical bone, the difference was 5 to $10 \%$, and this may have a clinical consequence and needs to be carefully considered in certain clinical situations.

\section{Abbreviations}

CTV: Clinical target volume; DD: Dose difference; $\mathrm{D}_{\mathrm{m}}$ : Dose to media; DTA: Distance to agreement; DVH: Dose volume histogram; $D_{w}$ : Dose to water; GTV: Gross tumor volume; HU: HOUNSFIELD unit; IMRT: Intensity modulated radiation therapy; MC: Monte carlo; MU: Monitor unit; NPC: Nasopharyngeal carcinoma; OAR: Organ at risk; PTV: Planning target volume; QA: Quality assurance; ROI: Region of interest; TPS: Treatment planning system

\section{Acknowledgements}

The authors would like to thank Dr. Yan Chen for many very helpful discussions.

\section{Availability of data and materials}

The datasets are backed up on the Research Data Deposit (RDD, http:// www.researchdata.org.cn, approval number: RDDA2017000317) and are available on reasonable request.

\section{Authors' contributions}

LC, BTH and XYH conceived and designed this study; LC and BTH wrote the manuscript with the help of XYH; WFC performed the data collective and dose calculations; WZS and XWD helped perform the analysis; All authors read and approved the final manuscript.

Ethics approval and consent to participate Not applicable.

\section{Consent for publication}

Not applicable.

Competing interests

The authors declare that they have no competing interests. 


\section{Publisher's Note}

Springer Nature remains neutral with regard to jurisdictional claims in published maps and institutional affiliations.

\section{Author details}

'Department of Radiation Oncology, Sun Yat-Sen University Cancer Center, State Key Laboratory of Oncology in South China, Collaborative Innovation Center for Cancer Medicine, Guangdong Key Laboratory of Nasopharyngeal Carcinoma Diagnosis and Therapy, No. 651, Dongfeng Road East, Guangzhou 510060, China. ${ }^{2}$ Department of Radiation Oncology, The first Affiliated Hospital of Sun Yat-Sen University, Guangzhou 510060, China.

Received: 27 January 2018 Accepted: 18 July 2018

Published online: 28 July 2018

\section{References}

1. International Commission on Radiation Units and Measurements (ICRU). ICRU Report No.33: Radiation quantities and units. Washington: ICRU; 1980.

2. Papanikolaou N, Battista J, Boyer A, Kappas C, Kein E, Mackie TR, Sharpe M, Dyk JV. AAPM Report No.85: Tissue inhomogeneity corrections for megavoltage photon beams. AAPM Report No. 85. Madison: Medical Physics Publishing; 2004. p. 1-135.

3. Fogliata A, Nicolini G, Vanetti E, Clivio A, Winkler P, Cozzi L. The impact of photon dose calculation algorithms on expected dose distributions in lungs under different respiratory phases. Phys Med Biol. 2008;53:2375-90.

4. Kathirvel M, Subramanian S, Clivio A, Arun G, Fogliata A, Nicolini G, Subramani V, Thirumalai Swamy S, Vanetti E, Cozzi L. Critical appraisal of the accuracy of Acuros-XB and anisotropic analytical algorithm compared to measurement and calculations with the compass system in the delivery of RapidArc clinical plans. Radiat Oncol. 2013;8(1):140.

5. Hartmann Siantar CL, Walling RS, Daly TP, Faddegon B, Albright N, Bergstrom P, Beilajew AF, Chuang C, Garrett D, RK H, Knapp D, Wieczorek DJ, Verhey LJ. Description and dosimetric verification of the PEREGRINE Monte Carlo dose calculation system for photon beams incident on a water phantom. Med Phys. 2001;28:1322-37.

6. Mika S, Christ G. Experimental validation of a Monte Carlo-based treatmentplanning system for Electron beams. Strahlenther Onkol. 2007;3:150-6.

7. Li J, Doemer A, Harrison AS, Galvin JM, Xiao Y. Dose calculation using Monte Carlo algorithm of Monaco treatment planning system. Int J of Radiat Oncol Bio Phys. 2008;72:S674.

8. Siebers JV, Keall PJ, Nahum AE, Mohan M. Converting absorbed dose to medium to absorbed dose to water for Monte Carlo based photon beam dose calculations. Phys Med Biol. 2000;45:983-95.

9. Liu H, Keall P. $D_{m}$ rather than $D_{w}$ should be used in Monte Carlo treatment planning. Med Phys. 2002:29:922-4.

10. Andreo P. Dose to 'water-like' media or dose to tissue in MV photons radiotherapy treatment planning: still a matter of debate. Phys Med Biol. 2015;60:309-37.

11. International Commission on Radiation Units and Measurements (ICRU). Report 50: Prescribing, recording, and reporting photon beam therapy. Bethesda: ICRU; 1993.

12. International Commission on Radiation Units and Measurements (ICRU). Report 62: Prescribing, recording and reporting photon beam therapy (supplement to ICRU report 50). Bethesda: ICRU; 1999.

13. International Commission on Radiation Units and Measurements (ICRU). Report 46: Photon, electron, proton and neutron interaction data for body issues. Bethesda: ICRU; 1992.

14. Fippel M. Fast Monte Carlo dose calculation for photon beams based on the VMC electron algorithm. Med Phys. 1999;26:1466-75.

15. International Commission on Radiation Units and Measurements (ICRU). Report 83: Prescribing, recording and reporting photon-beam IntensityModulated Radiation Therapy (IMRT). Journal of ICRU, 2010; https://doi.org/ 10.1093/jicru/ndq002.

16. Ma CM, Li J. Dose specification for radiation therapy: dose to water or dose to medium? Phys Med Biol. 2011;56:3073-89.

17. Fippel M, Nusslin F. Comments on ' converting absorbed dose to medium to absorbed dose to water for Monte Carlo based photon beam dose calculations.' Phys Med Biol. 2000;45:L17.

18. Chetty IJ, Curran B, Cygler JE, DeMarco JJ, Ezzel G, Faddegon BA, Kawrakow I, Keall PJ, Liu H, Ma CM, Rogers DW, Seuntjens J, Bagheri DS, Siebers JV.
Report of the AAPM Task Group No.105: Issues associated with clinical implementation of Monte Carl-based photon and electron external beam treatment planning. Med Phys. 2007;34:4818-53.

19. Dogam N , Siebers JV, Keall PJ. Clinical comparison of head and neck and prostate IMRT plans using absorbed dose to medium and absorbed dose to water. Phys Med Biol. 2006;51:4967-80.

20. Kan MW, Leung LH, So RW, Yu PK. Experimental verification of the Acuros $\mathrm{XB}$ and $\mathrm{AAA}$ dose calculation adjacent to heterogeneous media for IMRT and RapidArc of nasopharygeal carcinoma. Med Phys. 2013;40:031714. 1-19

\section{Ready to submit your research? Choose BMC and benefit from:}

- fast, convenient online submission

- thorough peer review by experienced researchers in your field

- rapid publication on acceptance

- support for research data, including large and complex data types

- gold Open Access which fosters wider collaboration and increased citations

- maximum visibility for your research: over $100 \mathrm{M}$ website views per year

At $\mathrm{BMC}$, research is always in progress.

Learn more biomedcentral.com/submissions 